\title{
A Novel Technology Combining RF and Magnetic Fields: Technical Elaboration on Novel RF Electrode Design
}

\author{
Diane Duncan*, M.D., FACS \\ Plastic Surgical Associates, Fort Collins CO, USA \\ *Corresponding author: Diane Duncan, M.D., FACS, Plastic Surgical Associates, Fort Collins CO, 1701 East Prospect Road, USA. \\ To Cite This Article: Diane Duncan, A Novel Technology Combining RF and Magnetic Fields: Technical Elaboration on Novel RF Electrode De- \\ sign. 2020 - 11(1). AJBSR.MS.ID.001608. DOI: 10.34297/AJBSR.2020.11.001608.
}

Received: 畊 November 28, 2020; Published: 制 December 08, 2020

\begin{abstract}
The article provides an insight into the technological aspects of a novel technology in body shaping allowing simultaneous delivery of HIFEM magnetic fields and radiofrequency energy. The device utilizes circular coil for magnetic field induction and radiofrequency electrodes in a single applicator which poses a technological challenge to the construction. To overcome the challenges a novel electrode design has been introduced for avoiding interferences between the two types of energy. As such the device presents a shift in the body contouring segment of aesthetic medicine, because it allows a simultaneous treatment of muscle laxity through supramaximal muscle stimulation and reduction of excessive adipose tissue through radiofrequency heating.
\end{abstract}

Keywords: HIFEM, Radiofrequency, Synchrode RF Electrode, Body Contouring

Abbreviations: HIFEM: High Intensity Focused Electromagnetic Field; RF: Radio Frequency

\section{Introduction}

In aesthetic medicine, various technologies are used for noninvasive body shaping. Two of the most widely used modalities are radiofrequency heating of tissues and high-intensity electromagnetic muscle activation. These two principles represent energy application arising from two different frequency bands of the electromagnetic spectrum within humans. Radio frequency (RF) are waves in the frequency range of roughly $20 \mathrm{kHz}$ to 300 $\mathrm{GHz}$. These are the frequencies with the ability to create heat through the oscillation of molecules while propagating within the tissue. In the aesthetic medicine, the RF energy is usually emitted by a solid metal electrode, which is normally in direct contact with the patient. The heating effects of the RF technology can be used for wide range of applications such as fat cell disruption, skin tightening or cellulite reduction [1-3]. For muscle activation, a highintensity electromagnetic field (HIFEM) is used. The HIFEM field represents a very-low frequency range $(<10 \mathrm{kHz})$.

During the application, rapid alternations in the magnetic field induce a secondary electric current in the tissue. These currents do not create any heat but are able to both polarize and depolarize the motor neurons that innervate the muscle, thus eliciting muscle contractions of supramaximal nature that cannot be achieved voluntarily. Such stimulation was found to result in muscle hypertrophy [4-6]. To emit HIFEM energy for muscle activation, an applicator with an embedded round metal coil is used without direct contact with the patient. Until recently, these two energies have been used solely as standalone modalities, and their combination in close-space engineering would be restricted by physical laws, due to frequency incompatibility and technical interference. However, the latest breakthrough in the field presented a technological solution overcoming these issues. The main goal of this article is thus to describe and explain the technical aspects of this innovation. Technical issues associated with simultaneous HIFEM and RF application In order to apply both RF and HIFEM at the same time within the same location, the metal RF electrode and the magnetic coil generating HIFEM field would need to be incorporated in a single applicator that is in contact with the patient.

However, while the magnetic fields easily pass through most common materials such as wood, plastic or even human tissue, 
they cannot pass through metal without mutual interactions. Any metals, when exposed to rapidly changing HIFEM fields quickly heat up (see Picture 1) and also tend to be physically repulsed. For this same reason, HIFEM is strictly contraindicated in patients with metal and electronic implants. With this known contraindication, HIFEM and ordinary RF can't be emitted simultaneously within the same field without overheating the metal electrode generating
$\mathrm{RF}$ as the electrodes are made of a solid metal. Although the RF electrodes are used to generate heat, their overheating could result in malfunctions in the RF delivery but most importantly would expose the patient to risk of burns. An evidence of the heating effect can be seen in the Figure 1 where metal plates are placed over the magnetic coil applicator (Figure 1).

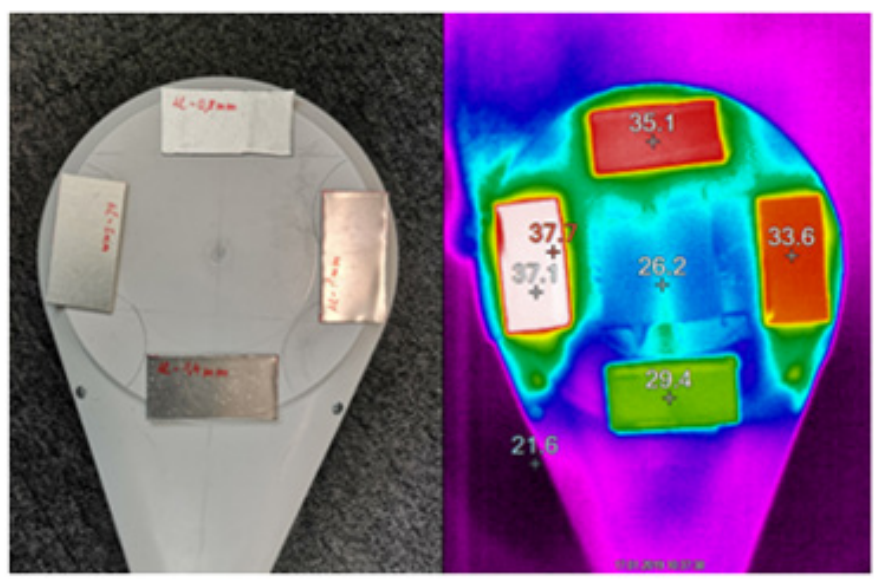

Figure 1: Digital photographs of running applicator containing the circular coil and metal plates placed over. The right image shows the metal plates' temperature elevation as captured by the thermal camera.

\section{Technical solution allowing simultaneous application}

To enable HIFEM to pass through the electrode without this heating phenomenon an entirely new electrode technology has been developed and patented by a leading company in the aesthetic market (BTL Industries Inc., Boston MA). While solid electrodes represent an issue for the magnetic field, the patented Synchrode RF electrode uses a special design concept which makes it transparent to the HIFEM energy. Instead of using a single coherent metal structure, the Synchrode RF electrode is comprised of 56 smaller segment pairs, with each segment pair laid out close to each other, creating individual positive and negative (+/-) RF poles (112 segments per applicator). The main purpose for using this segmented design and for having multiple interspacings is to achieve selective tissue heating effects as with large-sized solid electrode but without the technical limitations associated with a solid electrode (see Figure 2). The device utilizing this technology offers two treatment applicators, allowing a total of 224 interspaced electrode segments to work in synchrony during the treatment (Figure 2).
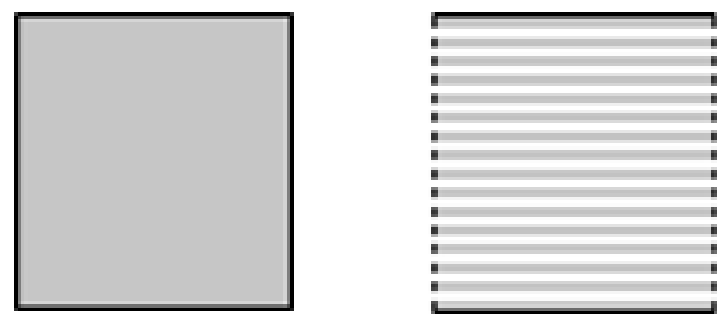

Figure 2: Illustration of the difference between standard and segmented electrode. Standard electrodes are made of compact material as a single piece (left), while the segmented electrode is composed of multiple interspaced segments (right). 
The key to this breakthrough technology is the interspacing. This interspaced design allows the HIFEM to pass through the electrode easily without any unfavorable interaction with its metal parts. Instead, the electrode is transparent to the magnetic fields, allowing them to continue in their intended direction (See Figure 3). Through years of development and testing, the Synchrode RF electrode's overall size and shape is specifically tailored to work in synchrony with the HIFEM magnetic fields. The electrode design takes into consideration the exact shape, the depth of penetration, as well as the intensity of HIFEM fields. The magnetic coil and the Sychrode RF electrode are thus truly synchronized with each other from an engineering standpoint (Figure 3).
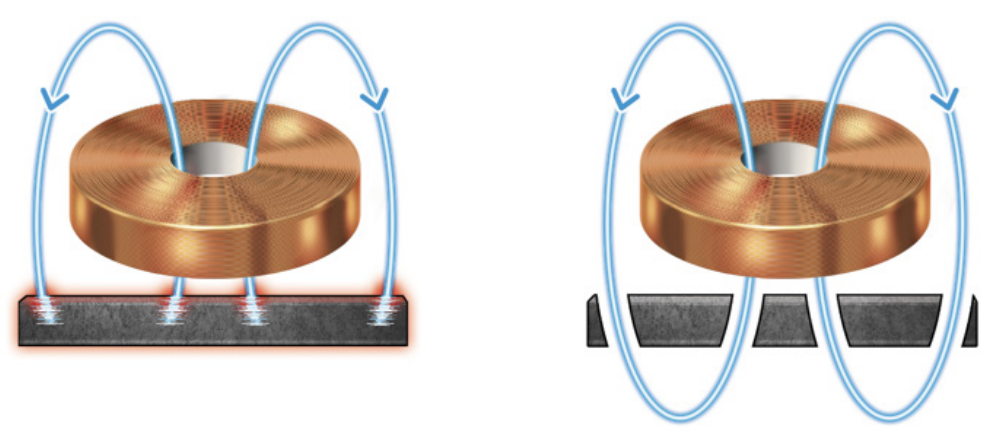

Figure 3: Illustration of the mutual interaction between magnetic coil and underlying electrode. The interspaced electrode is transparent to the HIFEM's magnetic field and the magnetic waves can pass without interaction, while standard electrode absorbs the HIFEM energy, resulting in heat.

\section{Conclusion}

For the very first time in the aesthetic field, this patented concept allows practitioners to simultaneously apply RF heating and highintensity electromagnetic muscle (HIFEM) activation in the same treatment area, at the same time. This represents an engineering breakthrough that now allows leveraging both the clinical, as well as commercial synergies of combining two gold standard technologies from a single device applicator. The company holding the patent rights (BTL Industries Inc., Boston MA) has already implemented this solution in a novel body shaping device introduced in October 2020 (EMSCULPT NEO) and presented the results of several clinical studies showing synergistic effects on muscle and fat tissues [710]. Although this electrode design solution has been first used for body shaping where it shows superior results, its' use may not be limited only to aesthetic medicine but could found a variety of other applications in another medical areas such as physiotherapy or rehabilitation.

\section{Conflict of Interest}

Dr. Diane Duncan is medical advisor for BTL, no funding was received for this article.

\section{References}

1. Angélica Rodrigues de Araújo, Viviane Pinheiro Campos Soares, Fernanda Souza da Silva, Tatiane da Silva Moreira (2015) Radiofrequency for the treatment of skin laxity: mith or truth. Anais Brasileiros de Dermatologia 90(5): 707-721.

2. Alizadeh Z, Halabchi F, Mazaheri R, Abolhasani M, Tabesh M, et al. (2016) Review of the Mechanisms and Effects of Noninvasive Body Contouring
Devices on Cellulite and Subcutaneous Fat. Int J Endocrinol Metab 14(4): e36727.

3. Emilia del Pino M, Rosado RH, Azuela A, Ma Graciela Guzmán, Dinorah Argüelles, et al. (2006) Effect of controlled volumetric tissue heating with radiofrequency on cellulite and the subcutaneous tissue of the buttocks and thighs. J Drugs Dermatol JDD 5(8): 714-722.

4. Duncan D, Dinev I (2019) Noninvasive Induction of Muscle Fiber Hypertrophy and Hyperplasia: Effects of High-Intensity Focused Electromagnetic Field Evaluated in an In-Vivo Porcine Model: A Pilot Study. Aesthet Surg J 40(5): 568-574.

5. Kinney BM, Lozanova P (2019) High intensity focused electromagnetic therapy evaluated by magnetic resonance imaging: Safety and efficacy study of a dual tissue effect based non-invasive abdominal body shaping. Lasers Surg Med 51(1): 40-46.

6. Kent DE, Jacob CI (2019) Simultaneous Changes in Abdominal Adipose and Muscle Tissues Following Treatments by High-Intensity Focused Electromagnetic (HIFEM) Technology-Based Device: Computed Tomography Evaluation. J Drugs Dermatol JDD 18(11): 1098-1102.

7. Weiss RA, Bernardy J, Tichy F (2020) Radiofrequency treatment used in combination with HIFEM therapy: Histological analysis includin scanning electron microscopy of adipocytes. Lasers Surg Med 52(S32): S5-S82.

8. Halaas Y, Bernardy J, Ondrackova P, Dinev I (2020) The skeletal muscle satellite cell activation by a combination of HIFEM procedure and radiofrequency treatment for body contouring: A first look at the NCAM/ CD56 facilitated detection by fluorescent microscopy. In: ASDS 2020

9. Katz BE, Samuels JB, Weiss RA (2020) Novel Radiofrequency Device Used in Combination with HIFEM Procedure for Abdominal Body Shaping: Sham-Controlled Randomized Trial. In: ASDS 2020.

10. Jacob C, Kent DE (2020) Abdominal Toning and Reduction of Subcutaneous Fat with Combination of HIFEM Procedure and Radiofrequency Treatment. In: ASDS 2020. 